\title{
Simple model for nonexponential relaxation in complex dynamics
}

\author{
A. Pérez-Madrid \\ Departament de Física, Fonamental, Facultat de Física, Universitat de Barcelona, Diagonal 647, Barcelona, Spain
}

(Received 27 November 2003; published 14 June 2004)

\begin{abstract}
The nonexponential relaxation occurring in complex dynamics manifested in a wide variety of systems is analyzed through a simple model of diffusion in phase space. It is found that the inability of the system to find its equilibrium state in any time scale becomes apparent in an effective temperature field, which leads to a hierarchy of relaxation times responsible for the slow relaxation phenomena.
\end{abstract}

DOI: 10.1103/PhysRevE.69.062102

PACS number(s): 05.40.-a, 05.10.Gg, 02.50.Ey, 64.70.Pf

Complex dynamics is the object of active research due to its implications in the technology of materials and in several fields of scientific knowledge. At the physicochemical and biological level, complex dynamics is observed in glassforming liquids; mechanical, dielectric, and magnetic relaxation; amorphous semiconductors; pinned density wave; protein dynamics; protein folding; and population dynamics among others. The mechanisms underlying slow relaxation in complex dynamics still lack a clear and definitive elucidation. In the case of supercooled liquids and glasses, several experiments and computer simulations have been done which support the explanation of these relaxation phenomena in the framework of the energy landscape paradigm as the result of activated diffusion through a rough energy landscape of valleys and peaks [1-4].

To understand what these mechanisms are, we propose here a simple model to show a possible origin of nonexponential relaxation based on the idea of the energy landscape and nontrivial energy barriers. This model, which consists of the diffusion in phase space, provides a direct link between the phase-space dynamics and the slow relaxation of the functions of the configuration of the system in the corresponding energy landscape. The slowing down of the dynamics clearly appears as a consequence of the freezing of some degrees of freedom, which takes the system out of equilibrium. This fact is indicated by the presence of an effective temperature field incorporating the information of the suppressed degrees of freedom and depending on the equilibrium temperature at the moment the quench was applied.

Hence, we model the relaxation in the liquid as the Brownian motion of a test particle of unit mass in a potential. As is well known, that physical situation is described by the Klein-Kramers equation, which for simplicity we write in one dimension,

$$
\frac{\partial}{\partial t} \hat{\rho}=-\frac{\partial}{\partial x} u \hat{\rho}+\frac{\partial}{\partial u} \hat{\rho} \frac{\partial}{\partial x} V(x)+\gamma \frac{\partial}{\partial u}\left(\beta^{-1} \frac{\partial}{\partial u}+u\right) \hat{\rho},
$$

where $\hat{\rho}(x, u, t)$ is the probability density, $x$ and $u$ are the position and velocity of the test particle, $\gamma$ its friction coefficient, and $\beta^{-1}=k_{B} T_{0}$, with $k_{B}$ being the Boltzmann constant and $T_{0}$ the bath temperature. Here, $V(x)$ is a nonperiodic potential constituting a schematic representation of a rough energy landscape. The local equilibrium solution for the
Kramers problem (1) is the Boltzmann distribution $\hat{\rho}_{\text {leq }}(x, u) \sim \exp \left\{-\beta\left[1 / 2 u^{2}+V(x)\right]\right\}$. Thus, inherent to Eq. (1) is the existence of local equilibrium in phase space $(x, u)$ $[5,6]$, and the approach to this state occurs at the bath temperature. Therefore, one concludes that at high temperatures the system possesses a large amount of energy to move through the whole phase space.

We assume that a quench of the system freezes the translational degrees of freedom taking the system away from equilibrium. Thus, this leads us to think of the partial decoupling of the probability density $\hat{\rho}(x, u, t)$

$$
\hat{\rho}(x, u, t)=\phi_{x}\left(u, t ; T_{0}\right) \rho(x, t),
$$

where the conditional probability $\phi_{x}\left(u, t ; T_{0}\right)$ describes a state of quasiequilibrium $[7,8]$ in which the system, unable to equilibrate at the bath temperature, remains hanging. Here, unlike for a soft potential, a wide distribution of barriers with very different sizes can be present. Therefore, it follows that the amplitude $\phi_{x}\left(u, t ; T_{0}\right)$ of the probability density $\hat{\rho}$ might depend on time and position. In fact, after quenching, the kinetic energy may be enough to overcome a small barrier but not a high-energy barrier in a different position, giving rise to an anomalous diffusion. Assuming that $u$ is the fast variable, one concludes that the dynamical processes in the system are associated to configurational changes related to $x$, which constitutes the slow variable whose probability density is $\rho(x, t)=\int \hat{\rho} d u$. Hence, by integration of Eq. (1) the time derivative of $\rho$ is obtained,

$$
\frac{\partial}{\partial t} \rho=-\frac{\partial}{\partial x} \int u \hat{\rho} d u
$$

which defines the current $J(x, t)=\int u \hat{\rho} d u$. This current evolves according to

$$
\begin{aligned}
\frac{\partial}{\partial t} J= & -\frac{\partial}{\partial x} \int u^{2} \hat{\rho} d u+\frac{\partial}{\partial x} V \int u \frac{\partial}{\partial u} \hat{\rho} d u \\
& +\gamma \int u \frac{\partial}{\partial u}\left(\beta^{-1} \frac{\partial}{\partial u}+u\right) \hat{\rho} d u,
\end{aligned}
$$

which has been obtained from Eq. (1). Then, after partial integration of Eq. (4) and using the decoupling approximation (2), for times $t \gg \gamma^{-1}$ we obtain 


$$
J(x, t)=-\tau\left\{\rho(x, t) \frac{\partial}{\partial x} V(x)+k_{B} \frac{\partial}{\partial x} \rho(x, t) T\left(x, t ; T_{0}\right)\right\},
$$

where $\tau=\gamma^{-1}$ and $T\left(x, t ; T_{0}\right)=\int u^{2} \phi_{x}\left(u, t ; T_{0}\right) d u$ is the second moment of the conditional distribution $\phi_{x}\left(u, t ; T_{0}\right)$, which plays the role of an effective temperature which contains information on the frozen degrees of freedom. After defining the effective potential $\Phi(x, t)=V(x)+k_{B} T\left(x, t ; T_{0}\right)$, Eq. (5) can be rewritten as

$$
J(x, t)=-D\left(x, t ; T_{0}\right) \frac{\partial}{\partial x} \rho(x, t)-\tau \rho(x, t) \frac{\partial}{\partial x} \Phi(x, t),
$$

where $D\left(x, t ; T_{0}\right)=\tau k_{B} T\left(x, t ; T_{0}\right)$ is the generalized diffusion coefficient. Thus, by substituting Eq. (6) into Eq. (3) and taking $\tau=1$ (i.e., rescaling the time $t$ ), this becomes the generalized diffusion equation

$$
\frac{\partial}{\partial t} \rho=\frac{\partial}{\partial x}\left\{D\left(x, t ; T_{0}\right) \frac{\partial}{\partial x} \rho(x, t)+\rho(x, t) \frac{\partial}{\partial x} \Phi(x, t)\right\} .
$$

Note that the temperature field $T\left(x, t ; T_{0}\right)$ introduces thermal barriers (or in others words nontrivial activation energies) in the system [3]. This effective temperature can be computed from Eq. (7) by using the fluctuation-dissipation theorem in the framework of linear-response theory; this was shown in two earlier papers $[8,9]$. The presence of these thermal barriers is a nonequilibrium effect that disappears when the system is in equilibrium. In addition, as a consequence of the elimination of degrees of freedom, the dynamics becomes non-Markovian and depends on the equilibrium temperature $T_{0}$ at the time of quench. By introducing the Massieu function

$$
\Theta(x, t)=\ln \rho(x, t)+\int \frac{1}{k_{B} T\left(x, t ; T_{0}\right)} \frac{\partial}{\partial x} \Phi(x, t) d x,
$$

the probability current Eq. (6) can be rewritten as

$$
J(x, t)=-D\left(x, t ; T_{0}\right) \rho_{\text {lqe }}(x, t) \frac{\partial}{\partial x} \exp \Theta(x, t),
$$

where

$$
\rho_{\text {lqe }}(x, t) \sim \exp \left\{-\int \frac{1}{k_{B} T(x, t)} \frac{\partial}{\partial x} \Phi(x, t) d x\right\}
$$

is the local quasiequilibrium probability density for which $J(x, t)=0$ or equivalently $\Theta(x, t)=$ const.

If in a point $x_{2}$ there is a sink where $\rho\left(x_{2}, t\right)=0$ and a source at $x_{1}$ so that a quasi-stationary current $J(t)$ can be established in the system, then integration of Eq. (9) gives us the quasistationary probability density

$$
\rho(x, t)=J(t) \rho_{\mathrm{lqe}}(x, t) \int_{x}^{x_{2}} \frac{d y}{D\left(y, t ; T_{0}\right) \rho_{\mathrm{lqe}}(y, t)} .
$$

On the other hand, a second integration leads to

$$
J(t)=n(t)\left(\int_{x_{1}}^{x_{b}} d x \rho_{\mathrm{lqe}}(x, t) \int_{x}^{x_{2}} \frac{d y}{D\left(y, t ; T_{0}\right) \rho_{\mathrm{lqe}}(y, t)}\right)^{-1},
$$

with $n(t)=\int_{x_{1}}^{x_{b}} \rho(x, t) d x$ being the population at the left of the barrier. Thus, we can formulate the following rate equation:

$$
\frac{d}{d t} n(t)=-K\left(t ; x_{b}\right) n(t),
$$

where

$$
K\left(t ; x_{b}\right)=\left(\int_{x_{1}}^{x_{b}} d x \rho_{\mathrm{lqe}}(x, t) \int_{x}^{x_{2}} \frac{d y}{D\left(y, t ; T_{0}\right) \rho_{\mathrm{lqe}}(y, t)}\right)^{-1}
$$

is the rate constant and $x_{b}$ is the position of the barrier between $x_{1}$ and $x_{2}$. The relaxation equation (12) admits the solution

$$
n(t)=n\left(t_{0}\right) \exp \left\{-\int_{t_{0}}^{t} K\left(t^{\prime} ; x_{b}\right) d t^{\prime}\right\} \equiv n\left(t_{0}\right) \exp \{-f(t)\}
$$

or

$$
\hat{n}(t)=\hat{n}\left(t_{0}\right) \exp \{-f(t / \tau)\},
$$

where $\hat{n}(t)=n(t / \tau)$ and the initial time $t_{0}$ is known in the literature as the waiting time. Both Eqs. (14) and (15) constitute our main result in the sense that we obtain a hierarchy of relaxation times starting from a Markovian equation, the Klein-Kramers equation describing Brownian motion in a field of force. Here, the function $f(t / \tau)$ might be interpreted as an algebraic function $(t / \tau)^{\beta}$ obtaining a stretched exponential behavior [10] or as a logarithmic function $-\alpha \ln A t$ which leads to a powerlaw behavior [11] that characterizes anomalous diffusion [12]. In addition, another reading of our result is possible since assuming the form of $f(t / \tau)$ is equivalent to assuming the form of the distribution of residence times $\psi(t) \equiv-(d / d t) n(t)$ [13]. Thus, an in-depth analysis of the implications of the supposition of a nonexponential distribution of residence times usually performed in continuoustime random-walk models reveals that this hypothesis might also be rooted in the nonequilibrium character of the dynamics inherent to the energy landscape picture as we have shown.

Our previous discussion applies to fragile glasses. In the case of strong glassy systems, the potential is almost periodic. Thus, when the temperature is lowered, any configurational change is preceded by an equilibration in momentum space given by a local Maxwellian. Hence, the activation energies are independent of the temperature but with a certain random spatial distribution. Averaging over this distribution of energy barrier gives one the corresponding relaxation $[4,14,15]$. In the literature there are other types of models undergoing nonexponential relaxation, such as, for example, disorder models [16]. 
We want to emphasize that the precise inputs of our model theory are the potential and the friction, which depends on the temperature. After a quench, the viscosity of the system increases dramatically and hence so does the friction. This leads to a freezing of the translational degrees of freedom, which are adiabatically eliminated.

We conclude that despite its simplicity, the model we study contains the main features observed in the dynamical slowing down observed in a wide variety of complex systems. Hence, we think this work will contribute to the understanding of some aspects of complex dynamics.

This work was supported by DGICYT of the Spanish Government under Grant No. PB2002-01267.
[1] P. G. Debenedetti and F. H. Stillinger, Nature (London) 410, 259 (2001).

[2] H. Frauenfelder, S. Sligar, and P. G. Wolynes, Science 254, 1598 (1991).

[3] S. Sastry, P. G. Debenedetti, and F. H. Stillinger, Nature (London) 393, 554 (1998).

[4] M. D. Ediger, Annu. Rev. Phys. Chem. 51, 99 (2000).

[5] J. M. Rubí and A. Pérez-Madrid, Physica A 264, 492 (1999).

[6] J. M.G. Vilar and J. M. Rubí, Proc. Natl. Acad. Sci. U.S.A. 98, 11081 (2001).

[7] S. Franz and M. A. Virasoro, J. Phys. A 33, 891 (2000).

[8] I. Santamaria-Holek, A. Perez-Madrid, and J. M. Rubi, J. Chem. Phys. 120, 2818 (2004).

[9] A. Pérez-Madrid, D. Reguera, and J. M. Rubí, Physica A 329,
357 (2003).

[10] C. A. Angell, K. L. Ngai, G. B. McKenna, P. F. McMillan, and S. W. Martin, J. Appl. Phys. 88, 3113 (2000).

[11] Hu Cang, Jie Li, V. N. Novikov, and M. D. Fayer, J. Chem. Phys. 118, 9303 (2003).

[12] R. Metzler and J. Klafter, Phys. Rep. 339, 1 (2000).

[13] C. F. Vardeman II and J. D. Gezelter, J. Phys. Chem. A 105, 2568 (2001).

[14] E. Rabani, J. D. Gezelter, and B. J. Berne, Phys. Rev. Lett. 82, 3649 (1999).

[15] X. Xia and P. G. Wolynes, Phys. Rev. Lett. 86, 5526 (2001).

[16] J. Klafter and M. F. Shlesinger, Proc. Natl. Acad. Sci. U.S.A. 83, 848 (1986). 